\title{
Systematic study of Langmuir films of different amino acid derivatives on several subphases
}

\author{
L. Románszki ${ }^{1, *}$ and $J$. Telegdi ${ }^{1,2}$ \\ ${ }^{1}$ Research Centre for Natural Sciences, Hungarian Academy of Sciences, Institute of Materials and Environmental \\ Chemistry, 1117 Budapest, Magyar tudósok körútja 2., Hungary \\ 2 Óbuda University, Faculty of Light Industry and Environmental Engineering, 1034 Budapest, Doberdó u. 6., Hungary
}

\begin{abstract}
In a Langmuir trough, we studied the formation and the behaviour upon compression of Langmuir films of 1) $\alpha$-hexadecyl derivative of tert-butoxycarbonylated glycine, respectively $\alpha$-octadecyl derivative of tert-butoxycarbonylated glycine methyl ester on $\mathrm{L}-$ and $\mathrm{D}$-amino acid subphases with/without $\mathrm{Cu}^{2+}$ ions, and 2) $N$-hexadecanoyl derivatives of four L-amino acids (Ala, Ser, Glu, and Phe) on pure water subphase. The films were characterized by their $A_{\mathrm{c}}$ and $A_{0}$ molecular areas, $\Pi_{\mathrm{c}}$ collapse pressure, $C^{-1}$ compression modulus, and the $\Pi_{C_{\max }^{-1}}$ surface pressure at maximum compression modulus. In this contribution, the found peculiarities of the different amphiphile-subphase pairs and the drawn conclusions about the structure of the films and the interactions of the amphiphiles with the subphases are presented.
\end{abstract}

\section{Introduction}

Langmuir (L) films and Langmuir-Blodgett (LB) layers of long side chain amino acid derivatives are interesting for the investigation of (bio)sensing mechanisms, drug delivery processes, 2D chiral organization and recognition, etc., and the development of sensor, corrosion inhibitor, anti-biofouling etc. layers. L films of Ala [1-3], Asp [4], Ser [5-6], Val [3] etc. alkyl derivatives have been studied on different subphases.

In our recent studies, we demonstrated the corrosion protection and antibiofouling ability of carboxylic and hydroxamic acid LB layers and self-assembled monolayers [7-12]. In the present study, the L films of two $\alpha$-alkyl amino acid derivatives and four $N$-hexadecanoyl amino acids on several subphases have been studied by surface pressure - area isotherms.

\section{Materials and methods}

The raceme mixtures of the two $\alpha$-alkyl amino acid derivatives, $\quad N$-( $t$-butoxycarbonyl)-2-hexadecyl-glycine methyl ester (BOC-GlyMe-C16) and $N$-( $t$-butoxycarbonyl)-2-octadecyl-glycine (BOC-Gly-C18), (see figure 1) have been synthesized as described in [13]. L-Nhexadecanoyl amino acids were prepared in a SchottenBaumann reaction from the respective L-amino acid and hexadecanoyl chloride. The resulting compounds were

\footnotetext{
* Corresponding author: romanszki.lorand@ttk.mta.hu
}

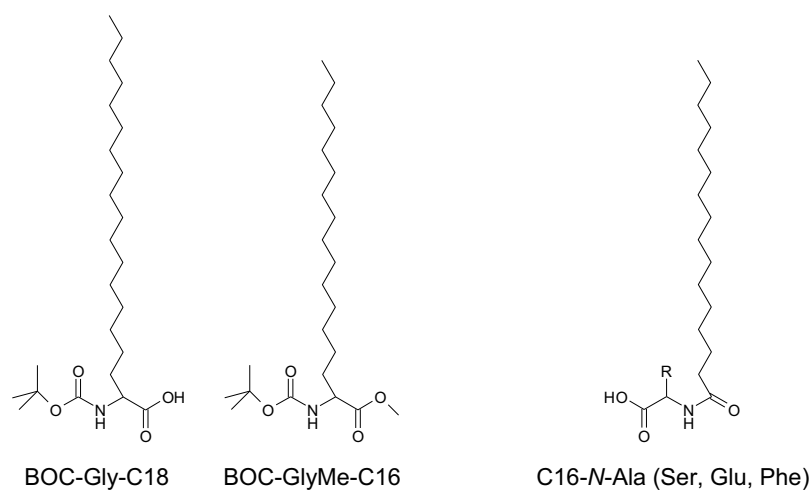

Fig. 1. Amphiphilic compounds used in these experiments: $\alpha$-alkyl amino acid derivatives BOC-Gly-C18 and BOCGlyMe-C16, respectively $N$-palmitoyl amino acids C16-N-Ala, C16-N-Ser, C16-N-Glu and C16-N-Phe.

purified in subsequent recrystallization steps, and were characterized by elemental analysis, melting point determination, thin layer chromatography and optical rotation. 30, 45 and $60 \mu \mathrm{l}$ volumes of $2 \mathrm{mM}$ solutions in chloroform were uniformly spread in a LB trough $(611 \mathrm{D}$, NIMA Technology Ltd., Coventry, UK) equipped with a Wilhelmy-type surface pressure gauge using filter paper. The $N$-hexadecanoyl amino acids were spread on a clear water subphase (MilliQ). The $\alpha$-alkyl amino acid derivatives were spread on: water, $1 \mathrm{mM} \mathrm{CuAc}_{2}, 100 \mathrm{mM}$ D-Ala, $100 \mathrm{mM}$ L-Ala, $100 \mathrm{mM}$ D-Asn, $100 \mathrm{mM} \mathrm{L-Asn}$, and mixed solutions containing $100 \mathrm{mM}$ D-Asn $+1 \mathrm{mM}$ $\mathrm{CuAc}_{2}$, respectively $100 \mathrm{mM} \mathrm{L-Asn}+1 \mathrm{mM} \mathrm{CuAc}$. 
Compression of the monolayers started 2 minutes after spreading, with a rate of $100 \mathrm{~cm}^{2} / \mathrm{min}$. The subphases were thermostated to $20^{\circ} \mathrm{C}$ with a precision of $0.5^{\circ} \mathrm{C}$. The obtained surface pressure - area isotherms were further processed to obtain collapse pressure, molecular areas and compression moduli.

\section{Results and discussion}

\section{1 $\alpha$-alkyl amino acid derivatives}

The isotherms of BOC-GlyMe-C16 and BOC-Gly-C18 on different subphases are shown in figure $2, \mathrm{~A}$ and $\mathrm{B}$. The derived compression modulus curves are presented in figure 2, C and $\mathrm{D}$. BOC-GlyMe-C16 shows a continuous increase of the surface pressure, going through an inflexion point, and then increasing with a mild slope without a breakdown. Contrarily, the isotherms of BOC-Gly-C18 initially start to increase with a moderate slope, go through a plateau region, and then rise steeply and finally a real collapse is observed.

The BAM images of the two amphiphile's monolayers (not shown) are accordingly very different. In the case of the BOC-GlyMe-C16 individual islands formed already at the moment of spreading with clear boundaries and high contrast can observed, whereas BOC-Gly-C18 spreads instantaneously and homogeneously on the surfaces forming continuous monolayers.

From the isotherms, the $A_{\mathrm{c}}$ molecular area at collapse and the corresponding $\Pi_{\mathrm{c}}$ collapse pressure are obtained. By extrapolation of the collapse point to zero surface pressure, the $A_{0}$ molecular area can be determined. Table 1 and figure 3 summarize these quantities. On each subphase 1) the collapse pressures of the BOC-Gly-C18 films are much higher; and 2) both molecular area values of the BOC-Gly-C18 films are much smaller than those of the BOC-GlyMe-C16 films.

The BOC-GlyMe-C16 monolayers collapse before reaching a true solid phase, continuously, the film layering up as the barrier moves. This collapse is very similar to the quasistatic collapse found at unsaturated fatty carboxylic and hydroxamic acid monolayers [7]. In contrast, the BOC-Gly-C18 films collapse instantaneously, after reaching a real solid phase.

These observations indicate that BOC-Gly-C18 molecules behave much more ideally than BOCGlyMe-C16 molecules. The BOC-Gly-C18 molecules interact with the water molecules through their free carboxylic groups. This interaction is apparently much stronger than the one among the film forming molecules themselves. Thus, the molecules spread readily and quickly on the water surface, covering the whole area uniformly. Contrarily, BOC-GlyMe-C16 molecules behave non-ideally. Probably due to the esterified carboxyl group, which is more hydrophobic than a free one, the molecules adopt distorted conformations with much larger space demand. They also tend to form aggregates from the very beginning of the spreading, the
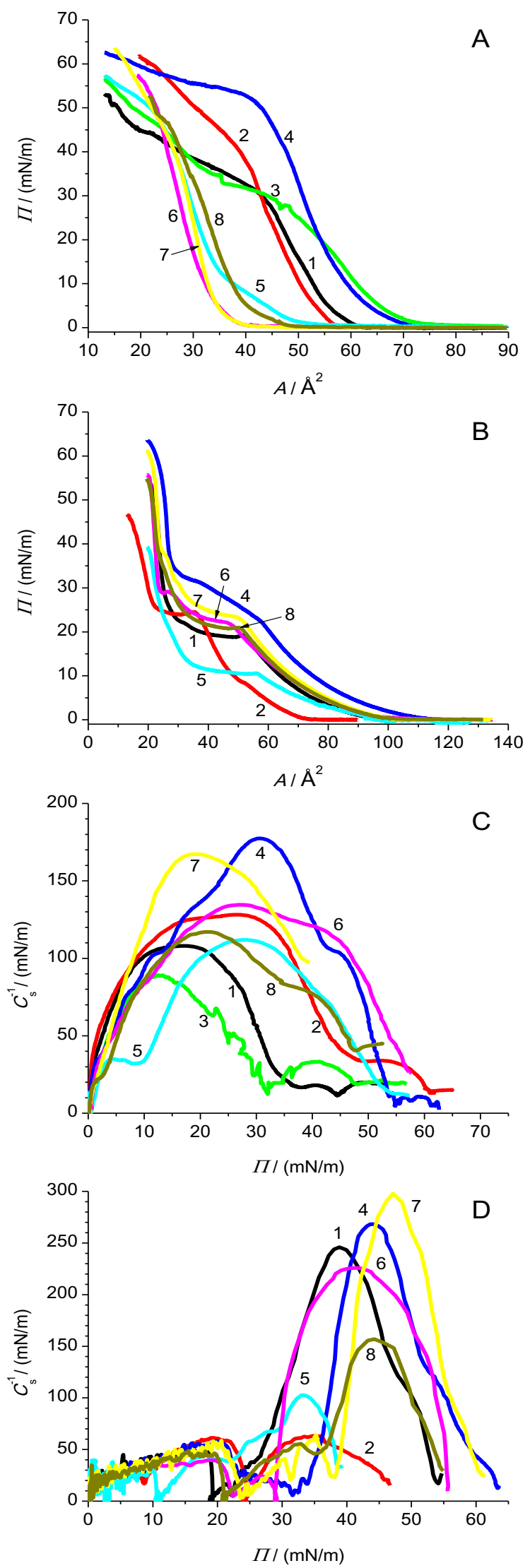

Fig. 2. Surface pressure $(\Pi)$ - molecular area $(A)$ isotherms of BOC-GlyMe-C16 (A) and BOC-Gly-C18 (B), and the corresponding compression modulus $\left(C_{\mathrm{s}}^{-1}\right)$ - surface pressure (II) curves $(\mathrm{C}$ and $\mathrm{D})$ at $20^{\circ} \mathrm{C}$ on different subphases: 1$)$ water; 2) $\mathrm{CuAc}_{2}$; 3) D-Ala; 4) L-Ala; 5) D-Asn; 6) D-Asn + $\mathrm{CuAc}_{2}$; 7) L-Asn; 8) L-Asn + $\mathrm{CuAc}_{2}$. 
interaction among the amphiphiles being stronger than the interaction between the amphiphiles and the water.

Table 1. $\Pi_{\mathrm{c}}$ collapse pressures as well as $A_{\mathrm{c}}$ and $A_{0}$ molecular areas of $\mathrm{L}$ monolayers of BOC-GlyMe-C16 and BOC-GlyC18 on different subphases. For each amphiphilic compound, the subphases with lowest and highest values are marked with light, respectively dark grey.

\begin{tabular}{|c|c|c|c|c|c|c|}
\hline \multirow{3}{*}{ \# subphase } & \multicolumn{3}{|c|}{ BOC-GlyMe-C16 } & \multicolumn{3}{|c|}{ BOC-Gly-C18 } \\
\hline & $\Pi_{\mathrm{c}}$ & $A_{\mathrm{c}}$ & $A_{0}$ & $\Pi_{\mathrm{c}}$ & $A_{\mathrm{c}}$ & $A_{0}$ \\
\hline & $\mathrm{mN} / \mathrm{m}$ & $\AA^{2}$ & $\AA^{2}$ & $\mathrm{mN} / \mathrm{m}$ & $\AA^{2}$ & $\AA^{2}$ \\
\hline 1 water & 19.3 & 48.3 & 50.8 & 38.0 & 23.9 & 27.1 \\
\hline $2 \mathrm{CuA}$ & 28.3 & 43.4 & 48.5 & 30.9 & 19.7 & 24.4 \\
\hline $3 \mathrm{D}-\mathrm{A}$ & 27.3 & 48.4 & 53.0 & & & \\
\hline $4 \mathrm{~L}$ & 29.7 & 51.0 & 57.1 & 42.4 & 26.3 & 29.1 \\
\hline $5 \mathrm{D}-\mathrm{A}$ & 31.6 & 28.8 & 35.4 & 32.6 & 21.9 & 27.1 \\
\hline $6 \mathrm{D}-\mathrm{Asn}+\mathrm{CuAc}_{2}$ & 28.1 & 27.6 & 32.1 & 42.7 & 22.0 & 25.4 \\
\hline 7 L-Asn & 27.0 & 29.4 & 33.8 & 44.8 & 23.2 & $26 .(1-x-1)$ \\
\hline $8 \mathrm{~L}-\mathrm{Asn}+\mathrm{CuAc}_{2}$ & 21.6 & 33.6 & 37.8 & 44.3 & 22.2 & 27. \\
\hline
\end{tabular}

Metal ions, as well as certain amino acids added to the subphase, are known to interact with the film forming molecules through strong coordinative and hydrogen bonds. The change in $\Pi_{\mathrm{c}}, A_{\mathrm{c}}$ and $A_{0}$ of these films with respect to those on pure water subphase, can be used to conclude about the strength and geometry of these bonds.

The copper ions in the subphase have, as expected, a compacting effect on the organization of the amphiphiles, decreasing both $A_{\mathrm{c}}$ and $A_{0}$. This tendency is more pronounced in the case of BOC-Gly-C18 than in the case of $\mathrm{BOC}-\mathrm{GlyMe}-\mathrm{C} 16$ that can be attributed to the presence of the methyl ester group in BOC-GlyMe-C16, as described above.

On both D- and L-Ala subphases, BOC-GlyMe-C16 monolayers exhibit slightly larger $A_{\mathrm{c}}$ and larger $A_{0}$ values than on pure water. Similarly, BOC-Gly-C18 monolayer on L-Ala subphase exhibits larger $A_{\mathrm{c}}$ and $A_{0}$ than on pure water. The $\Pi_{\mathrm{c}}$ collapse pressures are in each case higher on these amino acid subphases than on pure water subphases, indicating stiffer films. Thus Ala, in its both optical isomers, slightly expands the monolayers of these amphiphiles.
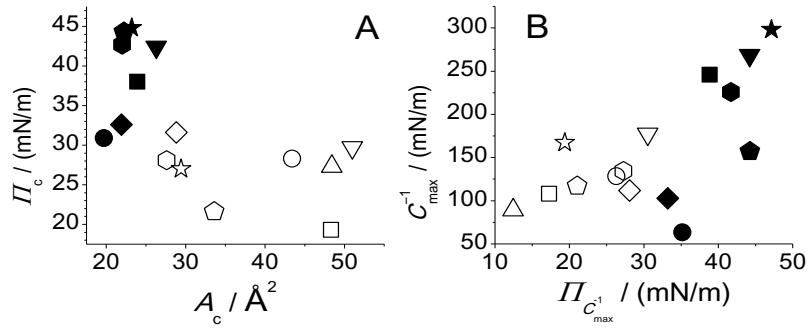

Fig. 3. $\Pi_{\mathrm{c}}$ collapse pressure and $A_{\mathrm{c}}$ molecular area values (A), respectively $C_{\max }^{-1}$ maximum compression moduli and the corresponding $\Pi_{\mathrm{C}_{\max }^{-1}}$ surface pressure values (B) determined from the $\mathrm{L}$ film isotherms of BOC-GlyMe-C16 (empty symbols), respectively BOC-Gly-C18 (filled symbols), recorded on different subphases: water $(\boldsymbol{\square})$; $\mathrm{CuAc}_{2}(\mathbf{O})$; D-Ala $(\boldsymbol{\Delta})$; L-Ala $(\boldsymbol{\nabla}) ; \quad$ D-Asn $(\bullet) ; \quad$ D-Asn $+\mathrm{CuAc}_{2}(\bullet) ; \quad$ L-Asn $(\star)$; $\mathrm{L}-\mathrm{Asn}+\mathrm{CuAc}_{2}(\bullet)$.

D-Asn, contrarily, has a close-packing effect: both BOC-GlyMe-C16 and BOC-Gly-C18 exhibit smaller molecular areas on D-Asn than on pure water. This packing effect is more pronounced in the case of BOCGlyMe-C16 than in the case of BOC-Gly-C18. For BOC-GlyMe-C16, it is even stronger than the effect of copper ions. By adding copper ions to the D-Asn subphase, the molecular areas decrease slightly even further. The effect of L-Asn is qualitatively similar and quantitatively very close to that of D-Asn: for BOCGlyMe-C16, $A_{\mathrm{c}}$ and $A_{0}$ are $39 \%$, respectively $33 \%$ smaller than on water, whereas for BOC-Gly-C18, by only $3 \%$, respectively $4 \%$. Interestingly, by adding copper ions to the L-Asn subphase, the molecular areas of the BOC-GlyMe-C16 monolayers decrease somewhat less as compared to the values on pure water. However, in the case of BOC-Gly-C18, $A_{\mathrm{c}}$ decreases slightly more on $\mathrm{L}-\mathrm{Asn}+\mathrm{CuAc}_{2}$ than on the pure L-Asn subphase, while $A_{0}$ practically does not change.

\section{2 $\mathrm{N}$-hexadecanoyl amino acids}

The isotherms and the derived compression modulus curves of the $N$-hexadecanoyl amino acid L films on pure water subphase are presented in figure 4 . Figure 5 shows the determined $\Pi_{\mathrm{c}}$ collapse pressures (A), $A_{\mathrm{c}}(\mathrm{B})$ and $A_{0}$ (C) molecular areas and the difference of these (D). In figure 6 , the position of the amphiphiles in a $\Pi_{\mathrm{c}}-A_{\mathrm{c}}$ coordinate system is presented.
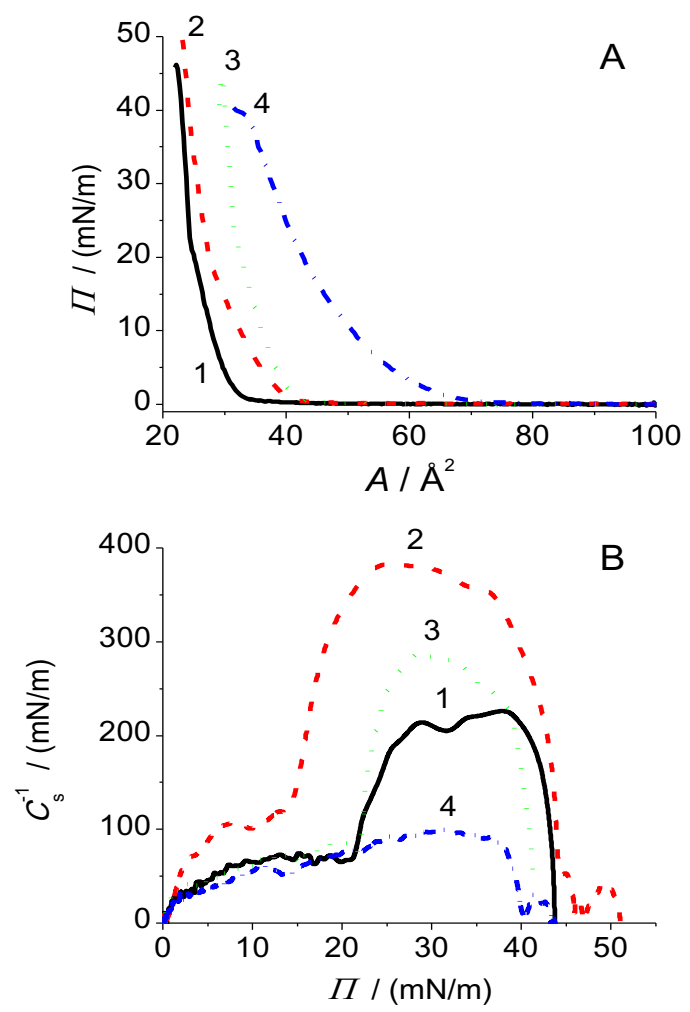

Fig. 4. Typical $\Pi-A$ isotherms (A) and the corresponding $C_{\mathrm{s}}^{-1}$ $\Pi$ curves (B) of the Ala (1), Ser (2), Glu (3) and Phe (4) C16amino acids.

The L films of the Ala and Ser derivatives exhibit almost identical molecular areas and collapse pressures, most likely due to the similarities in the molecular structures. The molecular areas of the Glu derivative L film are larger, with lower collapse pressure. The largest 
molecular area and the lowest collapse pressure were found in the case of the Phe derivative.

These results show strong correlation with the size of
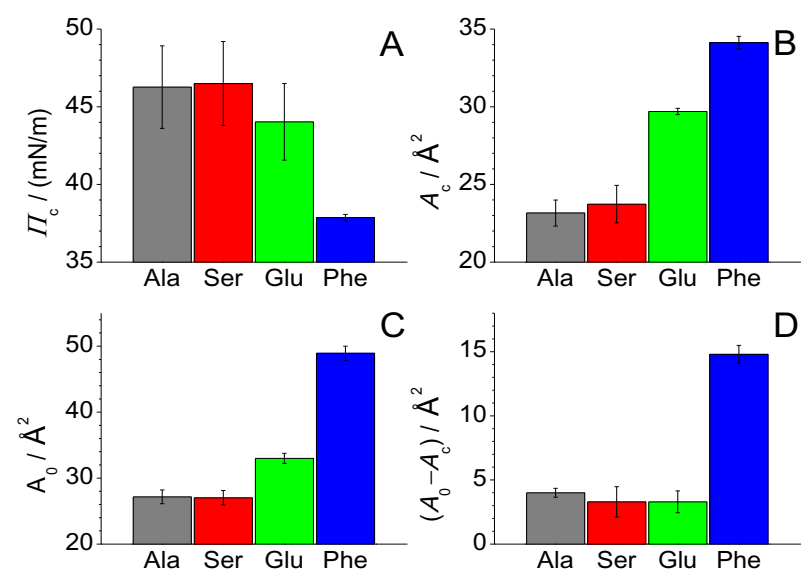

Fig. 5. $\Pi_{\mathrm{c}}$ collapse pressures (A), $A_{\mathrm{c}}$ molecular areas at collapse (B), $A_{0}$ molecular areas extrapolated to zero surface pressure (C) and the difference of the latter two (D) of the C16-amino acid derivatives $\mathrm{L}$ films.

the base amino acid (Ala $\approx$ Ser $<$ Glu $<$ Phe): with increasing amino acid size, the packing density of the $\mathrm{L}$ films is decreasing. Therefore it is expected that the most suitable compounds for densely packed, well ordered LB layer preparation, in about equal extent, are the $\mathrm{C} 16-\mathrm{N}-$ Ala and $\mathrm{C} 16-\mathrm{N}-\mathrm{Ser}$, due to the relatively small size of the amino acid part, and additionally, in the case of C16$\mathrm{N}-\mathrm{Ser}$, the hydrophilicity of the $\mathrm{OH}$ group. Contrarily, the large and hydrophobic phenyl group of $\mathrm{C} 16-\mathrm{N}-\mathrm{Phe}$ prevents molecules from tight packing, and its hydrophobicity does not favour an interaction with the water subphase.

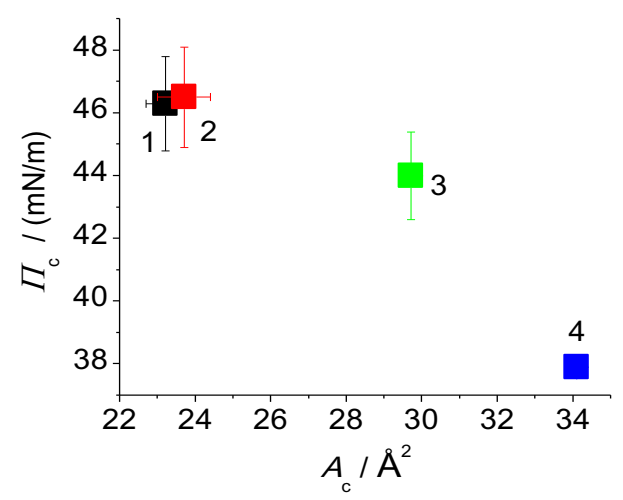

Fig. 6. The place of the Ala (1), Ser (2), Glu (3) and Phe (4) C16-amino acid derivative $\mathrm{L}$ films in a $\Pi_{\mathrm{c}}-A_{\mathrm{c}}$ coordinate system.

\section{Conclusions}

The presence or absence of a methyl ester group in the structure of the two $\alpha$-alkyl amino acid derivative amphiphiles causes significant differences in the organization of the monolayers at the subphase/air interface. $\mathrm{Cu}^{2+}$ ions in the water subphase have a closepacking effect on the monolayers of $\alpha$-alkyl amino acids. Different chiral amino acids in the subphase are interacting with the film-forming $\alpha$-alkyl amino acid molecules at the interface. The extent of this interaction depends on the interacting partners and needs further investigation. By selecting the adequate subphase, well ordered, closely packed LB layers can be prepared. The properties of the $\mathrm{L}$ films of $\mathrm{N}$-hexadecanoyl amino acids correlate with the size of the base amino acids. These results serve as good starting point for further investigations to clarify the role of the subphase and the strength of interaction between the $L$ film and the compounds in the subphase, as well as the effect of temperature and $\mathrm{pH}$. As the amino acids play important roles in transmembrane protein channels, we hope these answers would bring us closer to the development of new biosensing mechanisms.

\section{Acknowledgement}

The authors thank Katalin Tímár for the synthesis of the $\mathrm{N}$-alkanoyl amino acid derivatives.

\section{References}

1. O. Bouloussa, M. Dupeyrat, Biochim. Biophys. Acta 938, 395 (1988)

2. F. Hoffmann, H. Hühnerfuss, K.J. Stine, Langmuir 14, 4525 (1998)

3. F. Hoffmann, K.J. Stine, H. Hühnerfuss, J. Phys. Chem. B 109, 240 (2005)

4. K. Thirumoorthy, N. Nandi, D. Vollhardt, Colloid. Surface. A 282-283, 222 (2006)

5. N.G. Harvey, D. Mirajovsky, P.L. Rose, R. Verbiar, E.M. Arnett, J. Am. Chem. Soc. 111, 1115 (1989)

6. K.J. Stine, J.Y.J. Uang, S.D. Dingman, Langmuir 9, 2112 (1993)

7. L. Románszki, J. Telegdi, E. Kálmán, Colloid. Surface. A 321, 20 (2008)

8. L. Románszki, J. Telegdi, L. Nyikos, Stud. Univ. Babes-Bolyai Chem. 57, 191 (2012)

9. J. Telegdi, T. Szabó, L. Románszki, M. Pávai, In: Handbook of smart coatings for materials protection (Ed. ASH Makhlouf, Woodhead Publishing Ltd, Cambridge, 2014) 135

10. J. Telegdi, L. Románszki, F. Al-Taher, É. Pfeifer, E. Kálmán, In: $17^{\text {th }}$ International Corrosion Congress 2008: Corrosion Control in the Service of Society (NACE International, Houston, USA, 2009) 2631

11. L. Románszki, J. Telegdi, E. Kálmán, In: Proceedings of $6^{\text {th }}$ International Conference of PhD students. Natural Sciences. (University of Miskolc, Hungary, 2007) 97

12. J. Telegdi, F. Al-Taher, L. Románszki, E. Kálmán, In: The $3^{\text {rd }}$ International Conference on Technological Advances of Thin Films \& Surface Coatings (THINFILMS 2006) (Singapore, 2006) paper no. TCR640

13. W.A. Gibbons, R.A. Hughes, M. Charalambous, M. Christodoulou, A. Szeto, A.E. Aulabaugh, P. Mascagni, I. Toth, Liebigs Ann. Chem. 12, 1175 (1990) 